\title{
A Paradox Defined?
}

Recent decades have shown a shift in the global epidemiology of obesity. A problem historically related to material wealth, obesity afflicted mainly the developed world in the 20th century. Urbanization and a globalized food market have altered this association, and obesity is now considered a worldwide epidemic. ${ }^{1}$ Rates of end-organ disease such as coronary artery disease and ischemic stroke have risen in parallel with obesity rates. ${ }^{2}$ As populations age and the frequency of comorbid illness such as obesity, coronary disease, and stroke increase, demand for critical care services will surely rise. In the United States, the cost of critical care services amounts to an astonishing $1 \%$ of the gross domestic product. ${ }^{3}$ As the burden of critical illness rises throughout the world, economics will force a restructuring of care delivery. It is paramount to accurately describe the epidemiology of critically ill patients to better predict their outcomes and identify areas for improvement. It remains uncertain how obesity affects specific ICU outcomes.

Caring for obese patients in the ICU carries with it a unique set of challenges. Obese patients often have difficult airways, reduced ability to liberate from the ventilator, challenging central venous catheter placement, unreliable hemodynamic measurements, unreliable medication dosing and require labor-intensive nursing care. ${ }^{4}$ Given these management issues, one might expect poor ICU outcomes associated with obesity; however, this is still widely debated. Data from the medical literature is inconsistent. Multiple meta-analyses suggest a lower mortality rate among critically ill obese subjects, ${ }^{5-7}$ known as the obesity paradox. Oliveros and Villamor ${ }^{5}$ showed a lower risk of mortality among medical and surgical ICU subjects with a body mass index (BMI) between 18.5 and $24.9 \mathrm{~kg} / \mathrm{m}^{2}$ compared with those with a BMI between 30 and $39.9 \mathrm{~kg} / \mathrm{m}^{2}$. They found no associated mortality risk with a BMI of $\geq 40 \mathrm{~kg} / \mathrm{m}^{2}$. Akinnusi et $\mathrm{al}^{6}$ published another meta-analysis that compared the mortality of subjects with

\footnotetext{
Dr Shipe has disclosed no conflicts of interest.

Correspondence: Ryan G Shipe MD, Division of Pulmonary, Allergy, and Critical Care Medicine, Department of Medicine, University of Massachusetts Medical Center, 55 Lake Avenue North, Worcester, MA 01776. E-mail: ryan.shipe2@umassmemorial.org.
}

DOI: $10.4187 /$ respcare. 04243 a BMI of $>30 \mathrm{~kg} / \mathrm{m}^{2}$ with that of subjects with a BMI of $<30 \mathrm{~kg} / \mathrm{m}^{2}$. The authors found no difference in ICU mortality, but did find an improvement in mortality at the time of discharge. It is important to note that underweight subjects who were thought to have a higher mortality risk were included in the normal-weight groups in both of these studies, potentially biasing the results toward a higher mortality. Hogue et $\mathrm{al}^{7}$ excluded studies that included underweight subjects and found no association with obesity and better ICU survival, but did find a possible connection between better hospital survival and obesity. The results of each meta-analysis must be interpreted with caution because of the heterogeneity of the included studies. Despite these findings, little is known about the mortality of subgroups of critically ill obese patients.

\section{See the Original Study on Page 983}

In this issue of Respiratory CARE, Sasabuchi et al ${ }^{8}$ provide data to further delineate this phenomenon known as the obesity paradox by studying the effect of mechanical ventilation on outcomes of critically ill obese subjects. In their study, they compared the mortality of mechanically ventilated and non-mechanically ventilated subjects from multiple BMI subsets. Their data consist of the characteristics of 334,238 subjects from a Japanese national database. Of the subjects studied, $23.3 \%$ underwent mechanical ventilation within $2 \mathrm{~d}$ of admission. These ventilated subjects were more likely to suffer from sepsis, pneumonia, and coma. They were also more likely to undergo procedures such as blood product transfusion, catecholamine administration, and renal replacement therapy. Underweight subjects had the highest mortality rate, whereas overweight subjects had the lowest. Restricted cubic spline logistic regression analysis showed that non-obese mechanically ventilated subjects had a higher mortality rate compared with those who were not mechanically ventilated.

The study supports an obesity paradox in mechanically ventilated patients. It is a valuable addition to this subject for several reasons. The large number of subjects included allows small differences between groups to be determined, such as the difference Sasabuchi et al $^{8}$ documented between ventilated and non-ventilated subjects. Data representative of an entire nation's burden of illness is exceptional. Importantly, the study separates underweight 


\section{A Paradox Defined?}

subjects from other BMI categories and supports the idea that underweight patients have a worse prognosis in the ICU regardless of need for mechanical ventilation. Despite the study's attributes, more questions are raised than answered due to methodological concerns.

There are several limitations to the study, some of which were mentioned by the authors. ${ }^{8}$ The retrospective nature of the study disallowed randomization. Significant baseline differences among the subjects in each respective group were explained using statistical methods; however, the question must be raised whether the degree of heterogeneity can be corrected with statistical methods alone. Also, there was no information in the database regarding severity of illness. A possible explanation for a mortality benefit among the obese subjects who were mechanically ventilated is that they suffered from less severe illness at enrollment. This may not be the case, given that they were more likely afflicted with sepsis, pneumonia, and coma, but it remains unclear. It is very possible that these subjects had an acute reversible illness and baseline characteristics requiring them to undergo aggressive care such as mechanical ventilation. For example, little was known about chronic baseline comorbidities, which could have dissuaded providers from pursuing aggressive interventions, including mechanical ventilation. Additionally, subject preferences pertaining to goals of care were not documented. Either of these factors could easily account for a mortality benefit between subjects based on the need for mechanical ventilation. Finally, reasons for the initiation of mechanical ventilation were not included in the database. As mentioned previously, obese patients are prone to problems in the ICU, such as difficult airways, procedures, or increased need for nursing care. Obese patients with less severe acute illness may undergo mechanical ventilation for reasons related to their body habitus and not their severity of illness and may therefore have a better prognosis than normal-weight individuals who are intubated.

Despite findings in this and previous papers, the question of the obesity paradox persists. Moving forward, we must continue to ask ourselves if there are certain groups of patients, such as those who are mechanically ventilated, who have better outcomes, and if so, why? Some have proposed that the biology of anti-inflammatory molecules (eg, leptin) found in obese patients may be protective dur- ing bouts of critical illness. ${ }^{9}$ Others question whether obese patients receive higher levels of care because of a perceived poor prognosis. ${ }^{5}$ Future studies should differentiate extremes of weight (underweight, super obese) into separate categories to understand whether results are influenced by these potential confounders. A final question to raise is whether BMI is the correct variable to study in the first place. Obese patients have variable nutritional statuses and fitness levels, which are arguably more important to their physiologic response to critical illness. Gaining further understanding at a physiologic level may ultimately solve the question of the obesity paradox.

Ryan G Shipe MD

Division of Pulmonary, Allergy, and Critical Care

Medicine

Department of Medicine

University of Massachusetts Medical Center

Worcester, Massachusetts

\section{REFERENCES}

1. Caballero B. The global epidemic of obesity: an overview. Epidemiol Rev 2007;29(1):1-5.

2. Yatsuya H, Li Y, Hilawe EH, Ota A, Wang C, Chiang C, et al. Global trend in overweight and obesity and its association with cardiovascular disease incidence. Circ J 2014;78(12):2807-2818.

3. Kelly MA, Angus D, Chalfin DB, Crandall ED, Ingbar D, Johanson W, et al. The critical care crisis in the United States: a report from the profession. Chest 2004;125(4):1514-1517.

4. Honiden S, McArdle J. Obesity in the intensive care unit. Clin Chest Med 2009;30(3):581-599.

5. Oliveros H, Villamor E. Obesity and mortality in critically ill adults: a systematic review and meta-analysis. Obesity 2008;16(3):515-521.

6. Akinnusi ME, Pineda LA, El Solh AA. Effect of obesity on intensive care morbidity and mortality: a meta-analysis. Crit Care Med 2008; 36(1):151-158.

7. Hogue CW Jr, Stearns JD, Colantuoni E, Robinson KA, Stierer T, Mitter N, et al. The impact of obesity on outcomes after critical illness: a meta-analysis. Intensive Care Med 2009;35(7):1152-1170.

8. Sasabuchi Y, Yasunga H, Matsui H, Lefor AT, Horiguchi H, Fushimi $\mathrm{K}$, Sanui M. The dose-response relationship between body mass index and mortality in subjects admitted to the ICU with and without mechanical ventilation. Respir Care 2015;60(7):983-991.

9. Bornstein SR, Licinio J, Tauchnitz R, Engelmann L, Negrão AB, Gold P, Chrousos GP. Plasma leptin levels are increased in survivors of acute sepsis: associated loss of diurnal rhythm in cortisol and leptin secretion. J Clin Endocrinol Metab 1998;83(1):280-283. 